\title{
Accuracy of Ultrasound-Guided and Non-guided Botulinum Toxin Injection Into Neck Muscles Involved in Cervical Dystonia: A Cadaveric Study
}

\author{
Yun Dam Ko, MD¹, Soo In Yun, $\mathrm{MD}^{1}$, Dahye Ryoo, $\mathrm{MD}^{1}$, Myung Eun Chung, MD, PhD², Jihye Park, $\mathrm{MD}^{1}$
}

${ }^{1}$ Department of Rehabilitation Medicine, Eunpyeong St. Mary's Hospital, College of Medicine, The Catholic University of Korea, Seoul;
${ }^{2}$ Department of Rehabilitation Medicine, St. Paul's Hospital, College of Medicine, The Catholic University of Korea, Seoul, Korea

Objective To compare the accuracy of ultrasound-guided and non-guided botulinum toxin injections into the neck muscles involved in cervical dystonia.

Methods Two physicians examined six muscles (sternocleidomastoid, upper trapezius, levator scapulae, splenius capitis, scalenus anterior, and scalenus medius) from six fresh cadavers. Each physician injected ultrasoundguided and non-guided injections to each side of the cadaver's neck muscles, respectively. Each physician then dissected the other physician's injected muscle to identify the injection results. For each injection technique, different colored dyes were used. Dissection was performed to identify the results of the injections. The muscles were divided into two groups based on the difficulty of access: sternocleidomastoid and upper trapezius muscles (group A) and the levator scapulae, splenius capitis, scalenus anterior, and scalenus medius muscles (group B).

Results The ultrasound-guided and non-guided injection accuracies of the group B muscles were $95.8 \%$ and $54.2 \%$, respectively $(\mathrm{p}<0.001)$, while the ultrasound-guided and non-guided injection accuracies of the group A muscles were $100 \%$ and $79.2 \%$, respectively $(\mathrm{p}<0.05)$.

Conclusion Ultrasound-guided botulinum toxin injections into inaccessible neck muscles provide a higher degree of accuracy than non-guided injections. It may also be desirable to consider performing ultrasound-guided injections into accessible neck muscles.

Keywords Cervical dystonia, Ultrasonography, Botulinum toxins

\footnotetext{
Received December 11, 2019; Revised January 27, 2020; Accepted March 3, 2020; Published online September 28, 2020 Corresponding author: Jihye Park

Department of Rehabilitation Medicine, Eunpyeong St. Mary's Hospital, College of Medicine, The Catholic University of Korea, 1021 Tongil-ro, Eunpyeong-gu, Seoul 03312, Korea. Tel: +82-2-2030-2875, Fax: +82-2-2030-2876, E-mail: sophia@catholic.ac.kr

ORCID: Yun Dam Ko (https://orcid.org/0000-0003-0439-8063); Soo In Yun (https://orcid.org/0000-0002-5570-8407); Dahye Ryoo (https://orcid. org/0000-0002-9305-9030); Myung Eun Chung (https://orcid.org/0000-0002-7308-2815); Jihye Park (https://orcid.org/0000-0002-9764-128X).
}

(c) This is an open-access article distributed under the terms of the Creative Commons Attribution Non-Commercial License (http://creativecommons.org/ licenses/by-nc/4.0) which permits unrestricted noncommercial use, distribution, and reproduction in any medium, provided the original work is properly cited. Copyright $\odot 2020$ by Korean Academy of Rehabilitation Medicine 


\section{INTRODUCTION}

Cervical dystonia (CD) is a movement disorder characterized by neck muscle contractions that cause abnormal postures or movements that may involve tremor and pain. This disorder is extremely distressing and may have a negative impact on a patient's quality of life.

The treatment of CD is symptomatic. To improve the patient's quality of life, treatment should include physical therapy, oral medication, botulinum toxin (BoNT) injections, and surgical therapies [1]. The effect of physical therapy is often temporary. None of the large-scale double-blind and placebo-controlled studies have demonstrated its long-term effectiveness [2]. In the case of childhood-onset or combined dystonia, levodopa may be used first. However, there are currently no FDA (Food and Drug Administration)-approved oral medications. The systemic side effects should also be considered [3]. Surgery is usually selectively performed in patients who fail other less invasive treatments [1].

The current treatment guidelines recommend BoNT injections as the first-line treatment for $\mathrm{CD}$. In 2000, the FDA approved the application of BoNT for CD treatment [4-6]. Approximately 50\%-90\% of patients with CD experience symptomatic improvement with BoNT injections [7].

Conventionally, BoNT has been injected through clinical examination. Palpitation of target muscles, observation of muscle movement, knowledge of anatomical landmarks, and patient reporting of tenderness are also helpful. However, several studies have reported a low accuracy of needle placement when using only anatomical landmarks [8-10].

Neck muscles are small, thin, and close to each other. In addition, several vital vessels and nerves pass through the neck. Examples of these are the carotid artery, jugular veins, vagal nerve, and brachial plexus. Therefore, accurate muscular injections crucial $[11,12]$. In addition to direct injury to adjacent tissue, other common side effects of BoNT injection in the neck muscles include dysphagia and excessive muscle weakness [3]. The side effects caused by the drug itself depend mostly on local diffusion from the injection sites. Since the extent of diffusion is dose-dependent, the probability of side effects would be reduced correspondingly with BoNT dose reduction achievable by increasing the accuracy of injection [13].
Electromyography (EMG) is also a common technique used in targeting the muscles for BoNT injections. However, because the actions of some neck muscles are not clearly distinguished and co-contraction of adjacent muscles may be falsely attributed to a target muscle, physicians cannot verify if the EMG needle tip is located in the specific muscle without imaging guidance. Furthermore, other disadvantages of EMG-guided injection include increased discomfort due to the larger size of EMG needles and the inability to identify vital vessels and nerves [14].

Ultrasound allows fast, noninvasive, and real-time visualization of muscles and nearby structures. The needle position and overall BoNT injection procedure can be visualized, allowing accurate injection into target muscles and multiple sites along a single track.

Few publications have compared the accuracies of ultrasound-guided and non-guided injections into neck muscles $[15,16]$. In addition, some case reports and studies have used functional outcomes to assess accuracy. However, no studies have used direct visual confirmation.

This study was conducted to compare the accuracy of ultrasound-guided and non-guided BoNT injections into neck muscles through confirmation by cadaveric dissection.

\section{MATERIALS AND METHODS}

\section{Subjects}

This study examined six muscles (sternocleidomastoid, upper trapezius, levator scapulae, splenius capitis, scalenus anterior, and scalenus medius) as the targets of injection for each of the six fresh cadavers. No deformities and changes due to surgery or trauma were presented in the neck of the six cadavers.

\section{Methods}

Two physicians participated in this study. One was a specialist with more than 10 years of experience in physiology and ultrasound. The other was a physical medicine and rehabilitation resident with 2 years of training in musculoskeletal ultrasound. Each physician injected ultrasound-guided and non-guided injections to each side of the cadaver's neck muscles, respectively.

For each injection technique, different colored dyes 
were used. Dissection was performed to identify the accuracy of the injection. The dye, mixed with oil and acrylic paint, was injected into the target muscle using a gauge-16, 1.5-inch needle. The muscles were divided into two groups according to the depth of the muscles and difficulty of access: group A consisted of the sternocleidomastoid and upper trapezius muscles; group B consisted of the levator scapulae, splenius capitis, scalenus anterior, and scalenus medius muscles. The overall injection accuracies, the accuracy in each muscle, and the accuracy in each group were compared.

\section{Non-guided injection based on anatomical landmarks Sternocleidomastoid}

The sternocleidomastoid was injected at the level of the thyroid cartilage while rotating the head to the opposite side and grasping the medial and lateral borders of the muscle [17].

\section{Upper trapezius}

The upper trapezius was injected at $70 \%$ of the line distance from the most lateral point of the external occipital protuberance to the most lateral point of the acromion while grasping the anterior and posterior borders of the muscle [18].

\section{Levator scapulae}

The levator scapulae was injected $4 \mathrm{~cm}$ cephalad and 2 $\mathrm{cm}$ medial to the medial angle of the scapula [17].

\section{Splenius capitis}

The splenius capitis was injected at the apex of the posterior cervical triangle where it is readily accessible as a surface muscle [19].

\section{Scalenus anterior}

The scalenus anterior was injected in an anterior to posterior direction, $3 \mathrm{~cm}$ above the clavicle, through the lateral border of the sternocleidomastoid [19].

\section{Scalenus medius}

The scalenus medius was injected in a lateral to medial direction, $3 \mathrm{~cm}$ above the clavicle, posterior to the lateral border of the sternocleidomastoid [19].

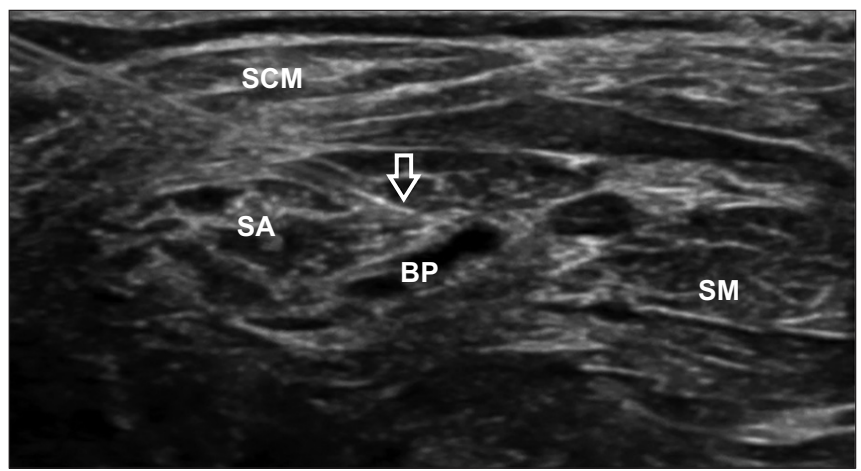

Fig. 1. Ultrasound-guided injection into the scalenus anterior (SA) muscle. Intramuscular needle (arrow) is visible in this figure. SCM, sternocleidomastoid; BP, brachial plexus; SM, scalenus medius.

\section{Ultrasound-guided dye injection}

Each muscle and the adjacent structures were identified under ultrasound guidance. A 3-12 MHz linear transducer (RS85; Samsung Medison, Seoul, Korea) was utilized for ultrasonography. The needle was inserted into the sternocleidomastoid, scalenus anterior, and scalenus medius in the supine position. For the trapezius, levator scapulae, and splenius capitis, the needle was inserted in the prone position. After confirming the position of the needle, dye was injected and simultaneously visualized through the ultrasound (Fig. 1).

\section{Sternocleidomastoid}

After the head was rotated to the opposite side and the probe was positioned longitudinal to the muscle fibers at the level of C6, the sternocleidomastoid was injected in a caudal to rostral direction using an in-plane technique $[11,20]$.

\section{Upper trapezius}

After the probe was positioned in the coronal plane midline between the spinous process of $\mathrm{C} 7$ and the acromion, the upper trapezius was injected in a medial to lateral direction using an in-plane technique $[11,20]$.

\section{Levator scapulae}

After the probe was positioned in the sagittal plane between the spinous process of $\mathrm{C} 7$ and the acromion while visualizing the superomedial border of the scapula on the screen, the levator scapulae was injected in a rostral to caudal direction using an in-plane technique through 
the upper trapezius $[11,20]$.

\section{Splenius capitis}

After the probe was positioned in the axial plane at the level of $\mathrm{C} 2$, the splenius capitis was injected in a medial to lateral direction using an in-plane technique $[11,14]$.

\section{Scalenus anterior}

After the head was rotated to the opposite side and the probe was positioned in the axial plane at the level of $\mathrm{C}$, the scalenus anterior was injected in a medial to lateral direction using an in-plane technique $[11,20]$.

\section{Scalenus medius}

After the head was rotated to the opposite side and the probe was positioned in the axial plane at the level of $\mathrm{C}$, the scalenus medius was injected in a lateral to medial direction using an in-plane technique [11].

\section{Assessment}

The physicians dissected the six cadavers from the other side of the injection and checked whether the dye was injected correctly into the target muscle (Fig. 2). The injection was considered to be "accurate" when the dye was visualized in the target muscle and "inaccurate" when it was not visualized in the target muscle.

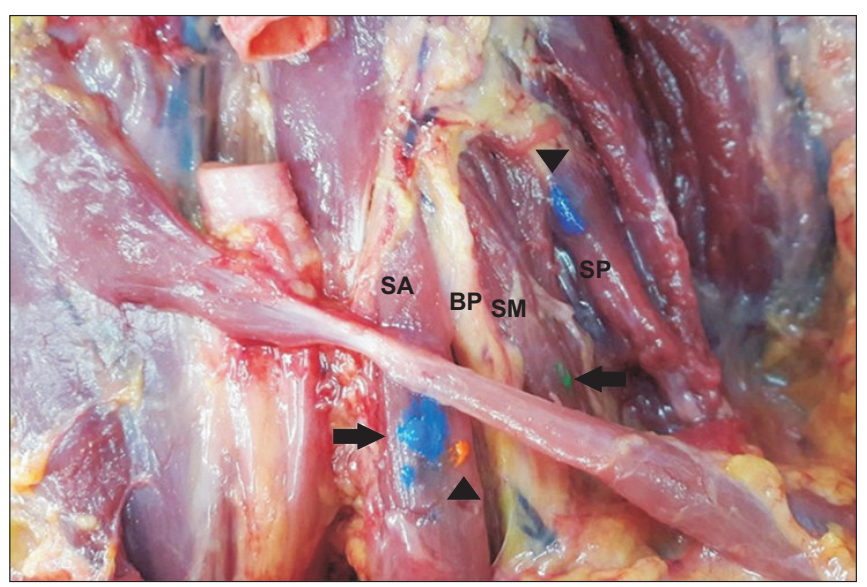

Fig. 2. Dissected cadaver with ultrasound-guided injection (blue and green dye, arrow) and non-guided injection (orange and purple dye, arrow head). The purple dye that targeted the scalenus medius muscle was misinjected into the scalenus posterior muscle. SA, scalene anterior; $\mathrm{BP}$, brachial plexus; SM, scalene medius; SP, scalene posterior.

\section{Statistical analysis}

All data analyses were computed by using the IBM SPSS version 23.0 software (IBM, Armonk, NY, USA). Fisher exact test was used to analyze the differences between the accuracies of the two injection techniques and the difference in the accuracy rates of the two physicians. The $\mathrm{p}$ values less than 0.05 were considered statistically significant.

\section{RESULTS}

The results of all injections are presented in Table 1 . The overall accuracies of the ultrasound-guided and non-guided injections into the six muscles were $97.2 \%$ and $62.5 \%$, respectively $(\mathrm{p}<0.001)$. However, there was variability in the injection accuracies in individual muscles. Specifically, the accuracies of the ultrasoundguided and non-guided injections were $100 \%$ and $83.3 \%$ ( $\mathrm{p}=0.478$ ) in the sternocleidomastoid muscle, $100 \%$ and $75.0 \%(\mathrm{p}=0.217)$ in the upper trapezius muscle, $91.7 \%$ and $50.0 \%(\mathrm{p}=0.069)$ in the levator scapulae muscle, $100 \%$ and $58.3 \%(\mathrm{p}=0.037)$ in the splenius capitis muscle, $91.7 \%$ and $58.3 \%(\mathrm{p}=0.155)$ in the scalenus anterior muscle, and $100 \%$ and $50.0 \%(\mathrm{p}=0.014)$ in the scalenus medius muscle, respectively.

The accuracies of the ultrasound-guided and non-guided injections into group B were $95.8 \%$ and $54.2 \%$, respectively $(p<0.001)$. The accuracies of the ultrasound-guided and non-guided injections into group A were $100 \%$ and $79.2 \%$, respectively $(\mathrm{p}<0.05)$. Between the two physicians, there was no significant difference between the accuracies of the ultrasound-guided injections $(p=0.305)$, the non-guided injections ( $\mathrm{p}=0.490)$, and the injections into groups $A$ and $B(p=1.000$ and $p=0.238)$.

\section{DISCUSSION}

The overall accuracies of the ultrasound-guided injections into the six neck muscles were higher than that of the non-guided injections $(\mathrm{p}<0.001)$. However, it is unreasonable to speculate that ultrasound-guided injections are better for all neck muscles since each muscle has a different level of access. To obtain more meaningful information, in this study, the muscles were divided into two groups according to the difficulty of access. In group $B$ muscles, the ultrasound-guided injections were sig- 
Table 1. Accuracy of ultrasound-guided and non-guided injections

\begin{tabular}{|c|c|c|c|c|c|}
\hline \multirow{2}{*}{ Muscles } & \multicolumn{2}{|c|}{ Non-guided } & \multicolumn{2}{|c|}{ Ultrasound-guided } & \multirow{2}{*}{ p-value } \\
\hline & Correct injection & Accuracy (\%) & Correct injection & Accuracy (\%) & \\
\hline \multicolumn{6}{|c|}{ Sternocleidomastoid } \\
\hline Physician_1 & $5(6)$ & 83.3 & $6(6)$ & 100 & \\
\hline Physician_2 & $5(6)$ & 83.3 & $6(6)$ & 100 & \\
\hline Total & $10(12)$ & 83.3 & $12(12)$ & 100 & 0.478 \\
\hline \multicolumn{6}{|l|}{ Upper trapezius } \\
\hline Physician_1 & $5(6)$ & 83.3 & $6(6)$ & 100 & \\
\hline Physician_2 & $4(6)$ & 66.7 & $6(6)$ & 100 & \\
\hline Total & $9(12)$ & 75.0 & $12(12)$ & 100 & 0.217 \\
\hline \multicolumn{6}{|l|}{ Levator scapulae } \\
\hline Physician_1 & $4(6)$ & 66.7 & $6(6)$ & 100 & \\
\hline Physician_2 & $2(6)$ & 33.3 & $5(6)$ & 83.3 & \\
\hline Total & $6(12)$ & 50.0 & $11(12)$ & 91.7 & 0.069 \\
\hline \multicolumn{6}{|l|}{ Splenius capitis } \\
\hline Physician_1 & $4(6)$ & 66.7 & $6(6)$ & 100 & \\
\hline Physician_2 & $3(6)$ & 50.0 & $6(6)$ & 100 & \\
\hline Total & $7(12)$ & 58.3 & $12(12)$ & 100 & 0.037 \\
\hline \multicolumn{6}{|l|}{ Scalenus anterior } \\
\hline Physician_1 & $3(6)$ & 50.0 & $6(6)$ & 100 & \\
\hline Physician_2 & $4(6)$ & 66.7 & $5(6)$ & 83.3 & \\
\hline Total & $7(12)$ & 58.3 & $11(12)$ & 91.7 & 0.155 \\
\hline \multicolumn{6}{|l|}{ Scalenus medius } \\
\hline Physician_1 & $4(6)$ & 66.7 & $6(6)$ & 100 & \\
\hline Physician_2 & $2(6)$ & 33.3 & $6(6)$ & 100 & \\
\hline Total & $6(12)$ & 50.0 & $12(12)$ & 100 & 0.014 \\
\hline Overall accuracy & $45(72)$ & 62.5 & $70(72)$ & 97.2 & $<0.001$ \\
\hline
\end{tabular}

Numbers in the parentheses indicate number of attempts.

The p-values were calculated as the difference between the accuracy of ultrasound-guided and non-guided injections.

nificantly more accurate than the non-guided injections $(\mathrm{p}<0.001)$. In group A muscles, both ultrasound-guided and non-guided injections were highly accurate. There was a significant difference between the two groups $(\mathrm{p}<0.05)$. Therefore, we found that ultrasound-guided injections may be desirable when injecting into the accessible-as well as the inaccessible-neck muscles.

The criteria for the selection of the six muscles in our study were determined from several studies. The most commonly injected muscles in patients with cervical dystonia were selected $[11,12,21]$. Because very inaccessible neck muscles such as the longus colli/capitis, longissimus capitis/cervicis, and obliquus capitis inferior are generally not injected without guidance in a clinical setting, these muscles were excluded. Furthermore, based on the anatomy of the deep cervical fascia, we divided the six muscles into two groups. Cervical fascia may be divided into the superficial and deep cervical fasciae. The deep cervical fascia consists of several layers. The investing layer that surrounds the sternocleidomastoid and trapezius muscles is the most superficial layer of the deep cervical fascia. The prevertebral layer is a deeper layer surrounding the deeper muscles of the neck. These include the prevertebral muscles, scalenus, levator scapulae, and splenius capitis muscles [22]. The exact location of the muscles categorized in group B were difficult to find using anatomical landmarks. Injections into these muscles carry a high risk of injecting neighboring muscles, nerves, or blood vessels. For example, errors in injections into the levator scapulae can result in injec- 
tions into the adjacent lung apex. The scalenus muscles are difficult to distinguish from each other. Injection into these muscles carries a risk of brachial plexus injury or external jugular vein puncture [12]. Therefore, when injecting these muscles, it is very important to inject in the correct location. Considering the results of this study, ultrasound guidance must be used for injections, especially on the inaccessible neck muscles.

Another consideration is that the usefulness of ultrasound guidance may vary depending on the difference in the proficiency of physicians. In our study, physician_2 had less clinical experience and tended to fail more injections than physician_1 under non-ultrasound guidance. The difference in injection accuracy between the two physicians tended to decrease under ultrasound guidance. However, the difference in the injection accuracy between the two physicians showed no statistically significant difference in all muscles-with or without ultrasound guidance. Further research may be needed to identify the differences in accuracy between physicians with more experience.

One of the strengths of this study is that we confirmed the injection results using anatomical verification with fresh cadavers rather than with functional outcomes. Functional outcomes such as the incidence of dysphagia can be influenced not only by the accuracy of the injection, but also by various factors such as the injection dose, muscle volume, and timing of evaluation. Therefore, the gold standard for determining the accuracy of any injection technique is an immediate post-injection surgical dissection in which the exact location of the injectate can be directly visualized [23]. Second, we divided the muscles into two groups according to the difficulty of access. For the application of imaging guidance in cervical dystonia, most previous studies have referred to only the inaccessible muscles with the assumption that imaging-guided injections will be more effective in these muscles $[15,24,25]$. These studies do not attempt to verify whether imaging-guided injections would also be more accurate for the accessible muscles. On the other hand, in addition to reaffirming the effectiveness of the ultrasound-guided injections in the inaccessible muscles, this study provides a clue that ultrasound-guided injections may also be desirable for the accessible muscles.

Our study also has several limitations. First, the sample size was small due to the limited number of cadavers available for the study. In the sternocleidomastoid and upper trapezius, the injection accuracies tended to be higher under ultrasound guidance $(100 \%$ and $83.3 \%$, and $100 \%$ and $75.0 \%$, respectively). However, these results were not statistically significant. Contrarily, when the two muscles were combined with the accessible muscles, there was a significant difference. It is thought that the statistical significance increased correspondingly with the sample size. The small sample size may also affect the results of the levator scapulae and the scalenus anterior. These muscles showed accuracies as low as the splenius capitis and scalenus medius muscles in non-guided injections. However, the statistical significance was not obtained by failing only once under ultrasound guidance. Therefore, this is a consideration in accepting the results of injections for each muscle. Additional samples are required to increase the reliability of statistical analysis. Second, observation of muscle movement or confirmation of tenderness would be possible if this study was performed on a living human. With the use of cadavers, the physical examination of the local injection site was limited. The skin and fat of the cadavers were harder and less distinguishable from the muscles. Therefore, the accuracy of non-guided injection may be underestimated compared to the actual clinical situation. Third, a gauge-16 needle was used for injection in this study. The needle had a larger diameter than those often used for BoNT injections into the neck muscle. In our study, a lager-diameter needle was chosen because it was difficult to inject viscous dyes with a small-diameter needle. Using a needle with a larger diameter allowed better visualization on the ultrasound. Therefore, this may have led to overestimation of the accuracy of the ultrasound-guided injections. Finally, we performed only quantitative analysis of the injection accuracy without a qualitative assessment. According to several reviews of BoNT injections, BoNT should be injected into the mid-portion of the muscle belly to allow homogenous distribution. This is possible with real-time visualization by ultrasound $[11,26]$. Further studies that can qualitatively assess the injections are needed. These may require identifying the difference in distance and depth between the injected dye and the midpoint of the muscle belly.

In conclusion, this study provides evidence that ultrasound-guided BoNT injections into the inaccessible neck muscles are more accurate than non-guided injections. 
In addition, we believe that it may also be desirable to consider performing ultrasound-guided injections into the accessible neck muscles.

\section{CONFLICT OF INTEREST}

No potential conflict of interest relevant to this article was reported.

\section{ACKNOWLEDGMENTS}

The authors would like to thank the Catholic Institute for Applied Anatomy, Department of Anatomy, College of Medicine, The Catholic University of Korea, Seoul, Korea, for its cooperation.

\section{AUTHOR CONTRIBUTION}

Conceptualization: Chung ME, Park J. Methodology: Ko YD, Park J. Formal analysis: Ko YD, Yun SI. Project administration: Ko YD, Park J. Visualization: Ko YD, Ryoo D. Writing - original draft: Ko YD. Writing - review and editing: Park J. Approval of final manuscript: all authors.

\section{REFERENCES}

1. Velickovic M, Benabou R, Brin MF. Cervical dystonia pathophysiology and treatment options. Drugs 2001;61:1921-43.

2. Van den Dool J, Visser B, Koelman JH, Engelbert RH, Tijssen MA. Long-term specialized physical therapy in cervical dystonia: outcomes of a randomized controlled trial. Arch Phys Med Rehabil 2019;100:1417-25.

3. Jinnah HA, Factor SA. Diagnosis and treatment of dystonia. Neurol Clin 2015;33:77-100.

4. Costa J, Espirito-Santo C, Borges A, Ferreira JJ, Coelho $\mathrm{M}$, Moore $\mathrm{P}$, et al. Botulinum toxin type A therapy for cervical dystonia. Cochrane Database Syst Rev 2005;(1):CD003633.

5. Hefter H, Benecke R, Erbguth F, Jost W, Reichel G, Wissel J. An open-label cohort study of the improvement of quality of life and pain in de novo cervical dystonia patients after injections with $500 \mathrm{U}$ botulinum toxin A (Dysport). BMJ Open 2013;3:e001853.

6. Skogseid IM, Malt UF, Roislien J, Kerty E. Determinants and status of quality of life after long-term botu- linum toxin therapy for cervical dystonia. Eur J Neurol 2007;14:1129-37.

7. Comella C, Bhatia K. An international survey of patients with cervical dystonia. J Neurol 2015;262:83748.

8. Henzel MK, Munin MC, Niyonkuru C, Skidmore ER, Weber DJ, Zafonte RD. Comparison of surface and ultrasound localization to identify forearm flexor muscles for botulinum toxin injections. PM R 2010;2:6426.

9. Schnitzler A, Roche N, Denormandie P, Lautridou C, Parratte B, Genet F. Manual needle placement: accuracy of botulinum toxin A injections. Muscle Nerve 2012;46:531-4.

10. Yang EJ, Rha DW, Yoo JK, Park ES. Accuracy of manual needle placement for gastrocnemius muscle in children with cerebral palsy checked against ultrasonography. Arch Phys Med Rehabil 2009;90:741-4.

11. Kaymak B, Kara M, Gurcay E, Ozcakar L. Sonographic guide for botulinum toxin injections of the neck muscles in cervical dystonia. Phys Med Rehabil Clin N Am 2018;29:105-23.

12. Schramm A, Baumer T, Fietzek U, Heitmann S, Walter U, Jost WH. Relevance of sonography for botulinum toxin treatment of cervical dystonia: an expert statement. J Neural Transm (Vienna) 2015;122:1457-63.

13. Brodsky MA, Swope DM, Grimes D. Diffusion of botulinum toxins. Tremor Other Hyperkinet Mov (N Y) 2012;2:tre-02-85-417-1.

14. Walter U, Dressler D. Ultrasound-guided botulinum toxin injections in neurology: technique, indications and future perspectives. Expert Rev Neurother 2014;14:923-36.

15. Fujimoto H, Mezaki T, Yokoe M, Mochizuki H. Sonographic guidance provides a low-risk approach to the longus colli muscle. Mov Disord 2012;27:928-9.

16. Hong JS, Sathe GG, Niyonkuru C, Munin MC. Elimination of dysphagia using ultrasound guidance for botulinum toxin injections in cervical dystonia. Muscle Nerve 2012;46:535-9.

17. Perotto AO. Anatomical guide for the electromyographer: the limbs and trunk. Springfield, IL: Charles C Thomas Publisher; 2011.

18. Lee JH, Lee KY, Kim JY, Son WH, Jeong JH, Gil Jeong $\mathrm{Y}$, et al. Botulinum toxin injection-site selection for a smooth shoulder line: an anatomical study. Biomed 
Res Int 2017;2017:3092720.

19. Truong D, Dressler D, Hallett M. Manual of botulinum toxin therapy. Cambridge, UK: Cambridge University Press; 2009.

20. Spinner DA, Kirschner JS, Herrera JE. Atlas of ultrasound guided musculoskeletal injections. New York, NY: Springer; 2014.

21. Yi YG, Kim K, Yi Y, Choi YA, Leigh JH, Bang MS. Botulinum toxin type A injection for cervical dystonia in adults with dyskinetic cerebral palsy. Toxins (Basel) 2018;10:203.

22. Sutcliffe P, Lasrado S. Anatomy, head and neck, deep cervical neck fascia [Internet]. Treasure Island, FL: StatPearls Publishing; 2020 [cited 2020 Aug 30]. Available from: https://www.ncbi.nlm.nih.gov/books/ NBK541091/.
23. Ziv YB, Kardosh R, Debi R, Backstein D, Safir O, Kosashvili Y. An inexpensive and accurate method for hip injections without the use of imaging. J Clin Rheumatol 2009;15:103-5.

24. Sung DH, Choi JY, Kim DH, Kim ES, Son YI, Cho YS, et al. Localization of dystonic muscles with 18F-FDG PET/CT in idiopathic cervical dystonia. J Nucl Med 2007;48:1790-5.

25. Lee IH, Yoon YC, Sung DH, Kwon JW, Jung JY. Initial experience with imaging-guided intramuscular botulinum toxin injection in patients with idiopathic cervical dystonia. AJR Am J Roentgenol 2009;192:996-1001.

26. Kaymak B, Kara M, Yagiz On A, Soylu AR, Ozcakar L. Innervation zone targeted botulinum toxin injections. Eur J Phys Rehabil Med 2018;54:100-9. 